\title{
Predicting structures and stabilities for H-type pseudoknots with interhelix loops
}

\author{
SONG CAO and SHI-JIE CHEN \\ Department of Physics and Department of Biochemistry, University of Missouri, Columbia, Missouri 65211, USA
}

\begin{abstract}
RNA pseudoknots play a critical role in RNA-related biology from the assembly of ribosome to the regulation of viral gene expression. A predictive model for pseudoknot structure and stability is essential for understanding and designing RNA structure and function. A previous statistical mechanical theory allows us to treat canonical H-type RNA pseudoknots that contain no intervening loop between the helices (see S. Cao and S.J. Chen [2006] in Nucleic Acids Research, Vol. 34; pp. 2634-2652). Biologically significant RNA pseudoknots often contain interhelix loops. Predicting the structure and stability for such moregeneral pseudoknots remains an unsolved problem. In the present study, we develop a predictive model for pseudoknots with interhelix loops. The model gives conformational entropy, stability, and the free-energy landscape from RNA sequences. The main features of this new model are the computation of the conformational entropy and folding free-energy base on the complete conformational ensemble and rigorous treatment for the excluded volume effects. Extensive tests for the structural predictions show overall good accuracy with average sensitivity and specificity equal to 0.91 and 0.91 , respectively. The theory developed here may be a solid starting point for first-principles modeling of more complex, larger RNAs.
\end{abstract}

Keywords: RNA folding; RNA pseudoknot; interhelix loop; structural predictions; folding thermodynamics

\section{INTRODUCTION}

An RNA pseudoknot is formed when nucleotides in a loop base-pair with complementary nucleotides outside the loop. An H-type pseudoknot is formed by base-pairing between a hairpin loop and the single-stranded region of the hairpin. The structure consists of two helix stems (Fig. $1 \mathrm{~A}, \mathrm{~S}_{1}, \mathrm{~S}_{2}$ ) and two loops (Fig. $1 \mathrm{~A}, \mathrm{~L}_{1}, \mathrm{~L}_{2}$ ) as well as a possible third loop/junction (Fig. $1 \mathrm{~A}, \mathrm{~L}_{3}$ ) that connects the two helix stems. In most naturally occurring RNA pseudoknots, interhelix loop $\mathrm{L}_{3}$ contains no more than 1 nucleotide (nt). For these canonical pseudoknot structures, helix stems $S_{1}$ and $S_{2}$ tend to stack coaxially (or partially coaxially) to form a quasicontinuous RNA helix in the three-dimensional space (3D) (Walter and Turner 1994; Chen et al. 1996; Cornish et al. 2005; Theimer et al. 2005). The coaxial stacking interaction can provide an essential stabilizing force for the structure.

The pseudoknot is a widespread motif in RNA structures (van Belkum et al. 1985; Perrotta and Been 1991; Tanner

Reprint requests to: Shi-Jie Chen, Department of Physics and Department of Biochemistry, University of Missouri, Columbia, MO 65211, USA; e-mail: chenshi@missouri.edu; fax: (573) 882-4195.

Article published online ahead of print. Article and publication date are at http://www.rnajournal.org/cgi/doi/10.1261/rna.1429009. et al. 1994; Deiman et al. 1997; Ferré-D’Amaré et al. 1998; Su et al. 1999; Schultes and Bartel 2000) and plays a variety of structural and functional roles in RNAs. For instance, pseudoknots form the core structural motif in the central catalytic domain of human telomerase RNA (Chen et al. 2000; Comolli et al. 2002; Theimer et al. 2005). As another example, for many viruses, pseudoknots play indispensable roles in promoting ribosomal frameshifting, a mechanism used by a retrovirus to regulate retroviral genome expression (Brierley et al. 1989, 2007; Somogyi et al. 1993; Giedroc et al. 2000; Plant et al. 2003; Staple and Butcher 2005; Namy et al. 2006; Hansen et al. 2007; Cao and Chen 2008; Pennell et al. 2008). Mutations that strengthen or weaken pseudoknot (thermal or mechanical) stability can cause changes in ribosomal frameshifting efficiency (Cornish et al. 2005; Theimer et al. 2005). For these, and a vast number of other RNA-related problems, quantitative prediction of pseudoknot structure and its stability is essential in order to unveil the mechanisms of RNA functions and in order to design therapeutic strategies for the diseases. In the present study, we develop a rigorous statistical mechanical model to predict the structure and folding stability for general RNA pseudoknots.

There are two main approaches used to predict RNA structures: free-energy minimization and comparative sequence 
A

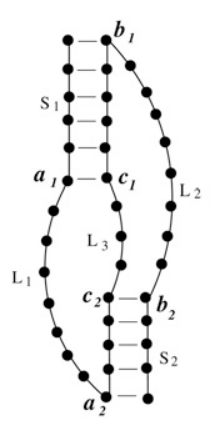

B

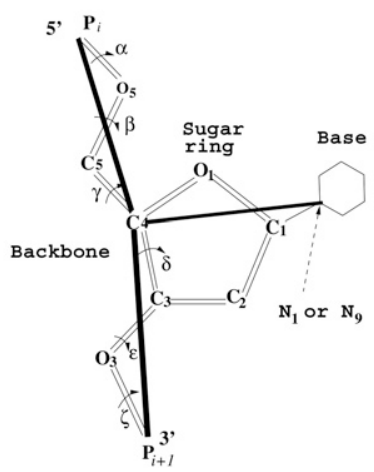

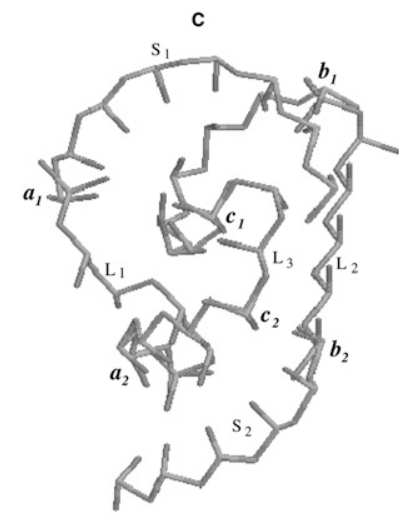

FIGURE 1. (A) An RNA pseudoknot with an interhelix loop $\mathrm{L}_{3}$. (B) Traditional two-vector virtual bond model for RNA nucleotides involves two bonds, $\mathrm{P}-\mathrm{C}_{4}-\mathrm{P}$. To describe the base orientation, we introduce a third virtual bond model, $\mathrm{C}_{4}-\mathrm{N}_{1}$ (pyrimidine) or $\mathrm{C}_{4}-\mathrm{N}_{9}$ (purine). (C) A virtual bond representation for a pseudoknot motif with $\mathrm{S}_{1}=8 \mathrm{bp}, \mathrm{S}_{2}=6 \mathrm{bp}, \mathrm{L}_{1}=4 \mathrm{nt}$, $\mathrm{L}_{2}=4 \mathrm{nt}$, and $\mathrm{L}_{3}=2 \mathrm{nt}$.

analysis. Existing free-energy-based algorithms are mainly for the prediction of the secondary structures. For instance, Nussinov and coworkers developed a dynamical programming algorithm for the prediction of the minimum freeenergy secondary structure (Nussinov et al. 1978; Nussinov and Jacobson 1980). Later, Williams and Tinoco (1986) extended the dynamical programming algorithm to find multiple low free-energy structures. In 1989, Zuker (1989) developed an advanced algorithm to predict all suboptimal low free-energy structures, and the algorithm led to the widely used Mfold software. In 1999, Mathews et al. (1999) developed an algorithm based on the much improved thermodynamic parameters. Algorithms based on the statistical mechanical partition function provide an alternative approach to predicting the structure and structural distributions (McCaskill 1990; Chen and Dill 2000; Hofacker 2003).

For RNA pseudoknots, several lines of computational algorithms have also been developed (Gultyaev et al. 1995; Rivas and Eddy 1999; Dirks and Pierce 2003; Reeder and Giegerich 2004; Ruan et al. 2004; Ren et al. 2005; Huang and Ali 2007; Chen et al. 2008; Metzler and Nebel 2008; Sperschneider and Datta 2008). Heuristic approaches (Ren et al. 2005) are computationally efficient, but unlike dynamic algorithms, they cannot guarantee finding the global free-energy minimum. Critical to an accurate structure prediction are the energy and entropy parameters. Current pseudoknot structural prediction algorithms often ignore the contribution of loop entropies (Ren et al. 2005) or use simplified (nonphysical) approximations (Dirks and Pierce 2003) for the loops. Although these models are remarkable in their computational efficiency to treat large RNA pseudoknots with hundreds of nucleotides (Mathews and Turner 2006; Reeder et al. 2006; Schuster 2006; Jossinet et al. 2007; Shapiro et al. 2007; Bon et al. 2008), their accuracies are limited by the availability of reliable param- free energy for simple canonical H-type pseudoknots (Cao and Chen 2006b), namely, pseudoknots with no interhelix loop (Fig. 1A, $\mathrm{L}_{3}$ ). For such canonical H-type pseudoknots, the two helix stems often form a quasicontinuous coaxially stacked helix. Central to the loop entropy calculation is the influence of the excluded volume between loop and helix. The effect of volume exclusion is sensitive to the stem and loop lengths. Here, we develop a new Vfold model to treat more complex pseudoknots that contain an interhelix loop (Fig. 1A, $\mathrm{L}_{3}$ ). The development of such a more-general model is significant for two reasons. First, the general pseudoknots studied here form the structural basis for large RNA folds, which involve multiple loops between helices. Second, the interhelix loops considered here are biologically important. For example, it has been suggested that a large class of anti-HIV RNA aptamers form pseudoknots with interhelix loops (Burke et al. 1996) so that the aptamers can be flexible and prevent the rigid coaxial stacking between the helices.

This paper is organized as follows. We first present a new three-vector virtual-bond-based RNA conformational model. The development of the new virtual bond model is motivated by the need to explicitly include the base orientations in addition to the backbone configuration considered in the original Vfold model (Cao and Chen 2005). We then use the new conformational model to compute the loop entropies in different pseudoknot contexts. A key issue in the calculation is how to account for the excluded volume effects. The entropy parameters will then allow us to predict the lowest free-energy structure as well as the folding thermodynamics from the RNA sequence. Comparisons with other models for structural prediction show improved results from our new model. As an application of the theory, we also investigate the equilibrium unfolding pathway for an anti-HIV RNA pseudoknot aptamer (Burke et al. 1996), the Visna-Maedi 
virus (VMV) pseudoknot (Pennell et al. 2008), and the 5' coding region of the R2 retrotransposon (Hart et al. 2008). The anti-HIV and VMV pseudoknots contain 3-nt and 6-nt interhelix loop $\mathrm{L}_{3}$, respectively.

\section{STRUCTURAL MODEL}

\section{A three-vector virtual bond model}

Because the torsional angles of the $\mathrm{C}-\mathrm{O}$ bonds (Fig. $1 \mathrm{~B}, \varepsilon, \beta$ ) in the nucleotide backbone tend to adopt the trans isomeric state, Olson (Olson and Flory 1972; Olson 1980) proposed to use a two-vector virtual bond to represent nucleic structures (see Fig. 1A). We recently developed a virtual-bond-based RNA folding model (the Vfold model) for H-type RNA pseudoknots (Cao and Chen 2006b). In Vfold, we model the helix as an A-form RNA helix using the experimentally measured atomic coordinates. For loops, which can be flexible, we use the usual gauche $e^{+}\left(g^{+}\right)$, trans $(t)$, and gauche $e^{-1}$ $\left(g^{-1}\right)$ rotational isomeric states for polymers (Flory 1969) to sample backbone conformations. The fact that the three isomeric states can be exactly configured in a diamond lattice (Cao and Chen 2005, 2006b) suggests that we can effectively configure the loop conformations as random walks of the virtual bonds on a diamond lattice. We note that the rotameric nature of RNA backbone conformations also has been observed for the known RNA structures (Duarte and Pyle 1998; Murthy et al. 1999; Murray et al. 2003; Wadley et al. 2007; Richardson et al. 2008).

The traditional two-vector virtual bond model cannot describe the base orientations. Motivated by the need to explicitly include base orientations in the structural description, we here propose a three-vector virtual bond model by introducing a third virtual bond to describe the base orientation (see Fig. 1B). Specifically, we add the $\mathrm{N}_{1}$ (for pyrimidine) or $\mathrm{N}_{9}$ (for purine) atom to the original $\mathrm{P}-$ $\mathrm{C}_{4}$ and $\mathrm{C}_{4}-\mathrm{P}$ virtual bonds (Fig. 1B). From the PDB database (Michiels et al. 2001; Theimer et al. 2005) for RNA pseudoknots, we find that the distance $\left(D_{\mathrm{CN}}\right)$ between $\mathrm{N}_{1}\left(\mathrm{~N}_{9}\right)$ and $\mathrm{C}_{4}$ atoms is close to $3.9 \AA$. In addition, we find that the torsion angle $(\chi)$ between plane $\mathrm{P}_{i}-\mathrm{C}_{4}-\mathrm{P}_{i+1}$ and plane $\mathrm{P}_{i}-\mathrm{C}_{4}-\mathrm{N}_{1}\left(\mathrm{~N}_{9}\right)$ is close to the $g^{-1}$ isomeric state; see the distributions for $D_{\mathrm{CN}}$ and the torsion angle in Figure 2. The localized distributions for the virtual bond $\mathrm{C}_{4}-\mathrm{N}_{1}\left(\mathrm{~N}_{9}\right)$ in Figure 2 suggest that $\mathrm{C}_{4}-\mathrm{N}_{1}\left(\mathrm{~N}_{9}\right)$ is quite rigid and can be configured in a diamond lattice. A previous study on RNA molecules also suggested a rigid base orientation (Olson and Flory 1972).

Figure 1A shows a pseudoknot with an interhelix loop. We use the atomic coordinates of the A-form RNA helix to configure the helices (Arnott and Hukins 1972). The $(r, \theta$, $z$ ) coordinates (in a cylindrical coordinate system) for the $\mathrm{P}, \mathrm{C}_{4}$, and $\mathrm{N}_{1}$ (or $\left.\mathrm{N}_{9}\right)$ atoms are $(8.71 \AA, 70.5+32.7 i$, $-3.75+2.81 i),(9.68 \AA, 46.9+32.7 i,-3.10+2.81 i)$, and $(7.12 \AA, 37.2+32.7 i,-1.39+2.81 i)(i=0,1,2, \ldots)$

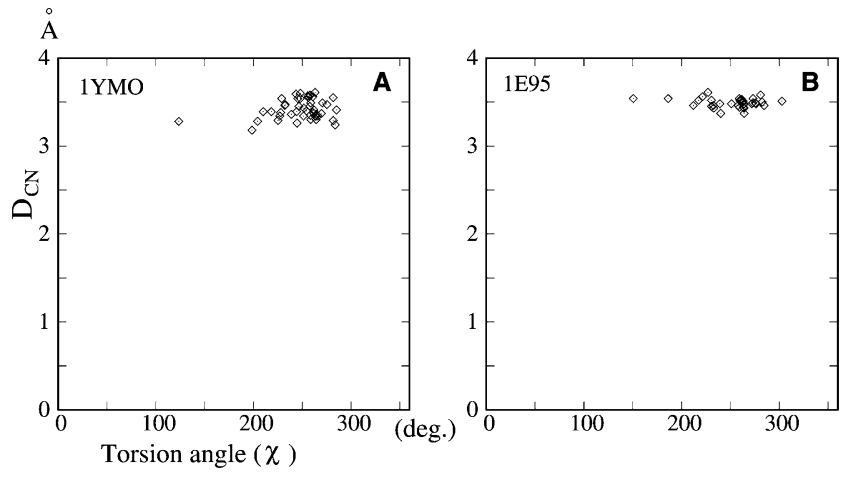

FIGURE 2. Survey of the $\left(D_{\mathrm{CN}}, \chi\right)$ distributions for two pseudoknot structures: $(A)$ the $47-n t \Delta U 177$ pseudoknot (Theimer et al. 2005) (PDB code: $1 \mathrm{YMO}$ ) and $(B)$ the 36-nt SRV-1 pseudoknot (Michiels et al. 2001) (PDB code: 1E95). $\mathrm{D}_{\mathrm{CN}}$ is the length of the $\mathrm{C}_{4}-\mathrm{N}_{1,9}$ virtual bond, and $\chi$ is the torsion angle between the $\mathrm{P}_{i}-\mathrm{C}_{4}-\mathrm{P}_{i+1}$ plane and the $\mathrm{P}_{i}-\mathrm{C}_{4}-\mathrm{N}_{1,9}$ plane.

(Arnott and Hukins 1972), respectively. For the other strand, we need to negate $\theta$ and $z$.

We generate loop $\left(\mathrm{L}_{1}, \mathrm{~L}_{2}\right.$, or $\left.\mathrm{L}_{3}\right)$ conformations through self-avoiding walks in a diamond lattice (Cao and Chen 2005), where a virtual bond is represented by a lattice bond. In the Vfold model, helices are configured off-lattice, while loop conformations are on-lattice. Loops and helices are connected via the six loop-helix interfacial nucleotides (Fig. 1A,C, $\left.a_{1}, a_{2}, b_{1}, b_{2}, c_{1}, c_{2}\right)$. These six interfacial nucleotides can be configured either as on-lattice loop terminals or as off-lattice helix terminals. We connect the loop and the helix through the minimum RMSD between the on-lattice and off-lattice coordinates for the six terminal nucleotides. Our calculation shows that the minimum RMSD is as small as $0.56 \AA$ for the $\left(\mathrm{P}_{i}, \mathrm{C}_{4}, \mathrm{~N}_{1}\right.$ or $\left.\mathrm{N}_{9}, \mathrm{P}_{i+1}\right)$ virtual bond atoms. The small RMSD indicates a smooth connection/transition between the on-lattice loop and the off-lattice helix.

\section{CONFORMATIONAL ENTROPY FOR PSEUDOKNOT WITH AN INTERHELIX LOOP}

For a given pseudoknot defined by the stem lengths $\left(S_{1}, S_{2}\right)$ and the loop lengths $\left(\mathrm{L}_{1}, \mathrm{~L}_{2}, \mathrm{~L}_{3}\right)$, we enumerate all the possible (virtual bond) conformations in the $3 \mathrm{D}$ space. From the total number of the viable conformations $\Omega$, we calculate the conformational entropy of the given pseudoknot as $\Delta \mathrm{S}\left(\mathrm{S}_{1}, \mathrm{~S}_{2}, \mathrm{~L}_{1}, \mathrm{~L}_{2}, \mathrm{~L}_{3}\right)=k_{\mathrm{B}} \ln \Omega$, where $k_{\mathrm{B}}=1.99 \mathrm{cal} /$ $\mathrm{K}$ is the Boltzmann constant. We choose different $\left(\mathrm{S}_{1}, \mathrm{~S}_{2}\right.$, $\mathrm{L}_{1}, \mathrm{~L}_{2}, \mathrm{~L}_{3}$ ) values (i.e., different pseudoknots), compute the conformational entropy for each pseudoknot, and compile the results as a large table for pseudoknot conformational entropy parameters.

Compared to simple canonical H-pseudoknots with no interhelix junction (junction-free pseudoknots), the pseudoknots here are much more complicated because the interhelix loop (Fig. 1, $\mathrm{L}_{3}$ ) between the two stems may be 

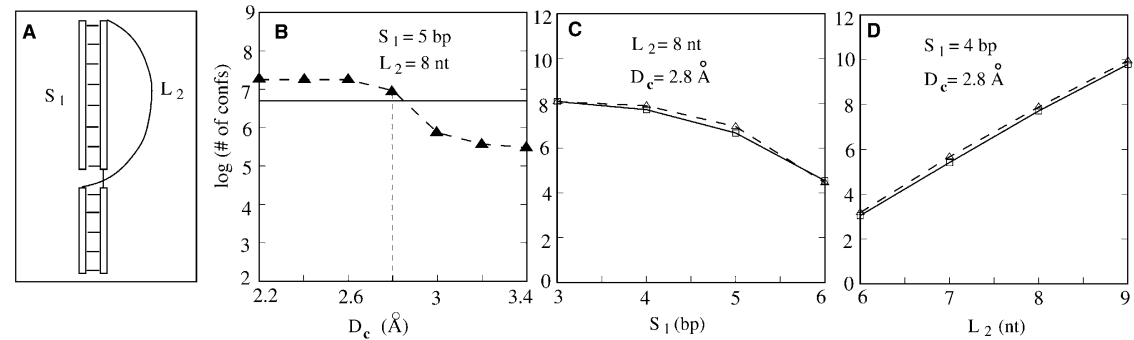

FIGURE 3. Test of the excluded volume effects with different distance cutoffs $\left(D_{c}\right)$. (A) Using a pseudoknot motif as the test system, we find that the cutoff distance of $2.8 \AA$ can best reproduce the conformational count from exact enumeration of on-lattice conformations. $(B)$ The triangles are the calculated entropies with cutoff distance varying from $2.2 \AA$ to $3.4 \AA$. The line denotes the entropies from the on-lattice exact computer enumeration. $(C)$ We also test the cutoff distances for different stem lengths while keeping the loop lengths fixed and $(D)$ for different loop lengths while keeping the stem length fixed. (Dashed lines) The results with offlattice helix and the cutoff distance; (solid lines) the results with on-lattice exact conformational enumerations. $(B-D)$ The $y$-axes are the numbers of loop $\left(\mathrm{L}_{2}\right)$ conformations (in log scale).

flexible, causing variable relative orientations between the helices. The previous model for the junction-free pseudoknots is a special case for the model developed here. To compute the total conformational count $\Omega$ for a pseudoknot with an interhelix loop, we enumerate the possible orientations between the two helices and then enumerate the loop conformations for each helix orientation:

$$
\Omega=\sum_{\text {helix orientation }} \Omega_{\mathrm{PK}},
$$

where $\Omega_{\mathrm{PK}}$ is the number of conformations for a given helix orientation.

\section{Enumeration of helix orientations}

The orientations of helices $S_{1}$ and $S_{2}$ are determined by the coordinates of the terminal nucleotides (Fig. 1A, $c_{1}, c_{2}$ ) of the loop $\mathrm{L}_{3}$. To enumerate the relative orientations of the helices, we fix the $\left(\mathrm{P}_{i}, \mathrm{C}_{4}, \mathrm{~N}_{1}\right.$ or $\left.\mathrm{N}_{9}, \mathrm{P}_{i+1}\right)$ coordinates for $c_{1}$, then enumerate the viable $\left(\mathrm{P}_{j}, \mathrm{C}_{4}, \mathrm{~N}_{1}\right.$ or $\left.\mathrm{N}_{9}, \mathrm{P}_{j+1}\right)$ coordinates for $c_{2}$. Specifically, we enumerate the loop $\mathrm{L}_{3}$ conformations as self-avoiding random walks of the virtual bonds in a diamond lattice. The number of possible coordinates of the terminal nucleotide $c_{2}$ (specifically, the coordinates of $\mathrm{P}_{j}, \mathrm{C}_{4}, \mathrm{~N}_{1}$, or $\mathrm{N}_{9}$, and $\mathrm{P}_{j+1}$ atoms of nucleotide $c_{2}$ ) is much smaller than the number of loop $\mathrm{L}_{3}$ conformations (see Fig. 4A, below). Therefore, the number of helix orientations, as determined by the $c_{1}$ and $c_{2}$ positions/coordinates, increases with $\mathrm{L}_{3}$ much more slowly than the number of loop $\left(\mathrm{L}_{3}\right)$ conformations. For instance, the number of helix orientations grows as $73 \rightarrow$ $390 \rightarrow 1358 \rightarrow 3208 \rightarrow 6096 \rightarrow 10,272 \rightarrow 15,984$ for an increasing interhelix loop length $1 \rightarrow 2 \rightarrow 3 \rightarrow 4 \rightarrow 5 \rightarrow$ $6 \rightarrow 7$ nt. The slow growth of the number of helix orientations makes the exact enumeration of all the possible helix orientations computationally viable.

\section{Enumeration of loop conformations for a given helix orientation}

For each relative orientation of the helices $S_{1}$ and $S_{2}$, we compute $\Omega_{\mathrm{PK}}$ in Equation 1 by enumerating the conformations for loops $\mathrm{L}_{1}$ and $\mathrm{L}_{2}$ and loop $\mathrm{L}_{3}$. The key is how to treat the excluded volume effect, i.e., the effect that different atoms cannot bump into each other.

We have two choices to treat the excluded volume effect here. We may fit the off-lattice helix onto the diamond lattice, then both helix and loop are configured in the same lattice, thus the volume exclusion effect can be conveniently treated in the lattice framework. Such an approach is computationally time-consuming because it requires offlattice $\rightarrow$ on-lattice fitting for all the helices for each and every helix orientation. Given the large number of helix orientations that we enumerate (Equation 1), the excluded volume treatment based on the above lattice-fitting is highly inefficient.

Alternatively, we can take a different approach that avoids the off-lattice $\rightarrow$ on-lattice fitting procedure. The strategy of the alternative approach is to keep the off-lattice helix structure. To treat the mixed system with the onlattice loop conformations and off-lattice helix structure, we introduce a cutoff distance $D_{c}$ such that atoms separated by a distance below the cutoff are considered to bump into each other, and the corresponding conformation is eliminated. Such a cutoff would allow us to treat the excluded volume effect in a unified framework, irrespective of the on-lattice or off-lattice representation of the conformations. We determine the value of the cutoff distance $D_{c}$ from the criterion that it gives the same entropy as the one calculated from the off-lattice $\rightarrow$ on-lattice fitting. We found that the optimal $D_{c}$ value is $2.8 \AA$ (see Fig. 3C,D). Therefore, in our entropy computation, we use $\mathrm{D}_{\mathrm{c}}=2.8 \AA$ as the criterion for volume exclusion.

For a fixed helix-helix orientation, we enumerate the loop conformations through self-avoiding random walks in the diamond lattice. The excluded volume between helix and helix, helix and loop, and loop and loop are explicitly considered. The treatment here for the excluded volume effect is more rigorous than previous Gaussian chain-based models (Gultyaev et al. 1999; Isambert and Siggia 2000; Bon et al. 2008), which ignore the excluded volume effect.

Using the three-vector virtual bond conformational model developed here, we can test the strengths of the different excluded volume effects (helix-helix, helix-loop, and loop-loop) (see Fig. 4B). We find that the loop-helix excluded volume interaction is strong. In contrast, the looploop excluded volume effect is rather weak (Fig. 4B), 

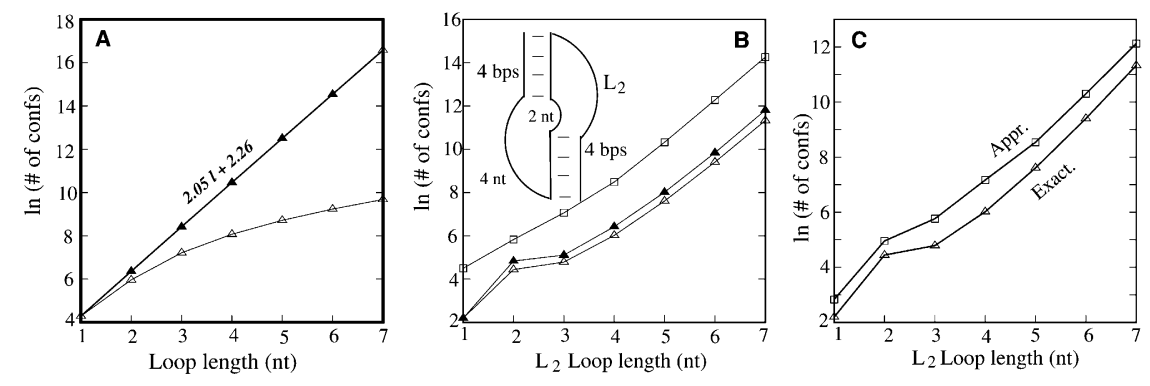

FIGURE 4. ( $A$, filled triangle) The number of single-stranded RNA chain conformations grows much faster than (unfilled triangle) the number of the end-end configurations (relative coordinates) of the chain. (B) We study the excluded volume effects on the loop entropy for a given pseudoknot with $\mathrm{S}_{1}=4 \mathrm{bp}, \mathrm{S}_{2}=4 \mathrm{bp}, \mathrm{L}_{1}=4 \mathrm{nt}$, and $\mathrm{L}_{3}=2 \mathrm{nt}$. We vary the loop length for $\mathrm{L}_{2}$ from $1 \mathrm{nt}$ to $7 \mathrm{nt}$ and find that we can neglect the loop-loop excluded volume interactions. (Open square) Results without excluded volume interactions; (filled triangle) results without considering the loop-loop excluded volume interactions; (open triangle) results with all the excluded volume interactions fully considered. (C) Comparison between the conformational entropy from the exact computer enumeration and the entropy from our theory. The deviation is small ( $<1 k_{\mathrm{B}} T$ in free energy).

suggesting that we can neglect loop-loop correlations when enumerating the conformations. This leads to the "independent loop approximation": we can ignore the presence of the other loops when we count the conformations for a loop.

Tests for the above method against exact computer enumeration suggest that our method can give reliable conformational entropies (see Fig. 4C). We have computed and tabulated the loop entropies $\Delta \mathrm{S}\left(\mathrm{S}_{1}, \mathrm{~S}_{2}, \mathrm{~L}_{1}, \mathrm{~L}_{2}, \mathrm{~L}_{3}\right)$ for stems $\mathrm{S}_{1}$ and $\mathrm{S}_{2}$ as $\leq 10 \mathrm{bp}$; loops $\mathrm{L}_{1}, \mathrm{~L}_{2}$ as $\leq 7 \mathrm{nt}$; and loop $\mathrm{L}_{3}$ as between $2 \mathrm{nt}$ and $6 \mathrm{nt}$. For $\mathrm{L}_{3} \leq 1 \mathrm{nt}$, we use the entropy table in Cao and Chen (2006b). The results for $\Delta \mathrm{S}\left(\mathrm{S}_{1}, \mathrm{~S}_{2}, \mathrm{~L}_{1}, \mathrm{~L}_{2}, \mathrm{~L}_{3}\right)$ are compiled as an entropy table (see Supplemental Table S1). On a Dell EM64T cluster system with $48 \operatorname{Intel}(\mathrm{R}) \mathrm{Xeon}(\mathrm{R}) 5150(2.66 \mathrm{GHz})$ processors, the computation of the full entropy table took less than a week.

For large loops $\mathrm{L}_{1}>7 \mathrm{nt}$ or $\mathrm{L}_{2}>7 \mathrm{nt}$, we use the following fitted formula (Serra and Turner 1995) for the entropy $\Delta S$ :

$$
\Delta S / k_{B}=a \log (l)+b,
$$

where $l$ is the loop length and $a$ and $b$ are fitted parameters; see Supplemental Tables S2 and S3 for the $a$ and $b$ parameters for different loop sizes.

\section{PARTITION FUNCTION}

In this section, we show how to use the recursive algorithm (Cao and Chen 2005, 2006b) to compute the partition function, from which all the thermodynamic properties of the system can be determined. Our partition function calculation is a sum over all the possible secondary and pseudoknotted structures, with and without an interhelix loop. A typical "prototype" structure contains internal/ bulge loops in the helix stems (see Fig. 5). Our conformational ensemble also includes other structures that stem from the prototype structure. For example, for the pseudoknot in Figure 5, we enumerate different $\mathrm{L}_{1}$ and $\mathrm{L}_{2}$ loop structures by allowing the formation of possible secondary structures in loops $\mathrm{L}_{1}$ and $\mathrm{L}_{2}$. We also allow multidomained structures, where each domain is an independently folded pseudoknotted or secondary structure.

We denote the partition function for the ensemble of all the possible pseudoknotted structures by $\mathrm{C}_{3}(a, b)$, where $a$ and $b$ denote the $5^{\prime}$ and $3^{\prime}$ terminal nucleotides, respectively, and the subscript 3 denotes a pseudoknot (tertiary) structure. A general pseudoknotted structure shown in Figure 5 is described by 11 parameters: the lengths of loops $\left(\mathrm{L}_{1}, \mathrm{~L}_{2}, \mathrm{~L}_{3}\right)$, the lengths of upper and lower segments of the helices $\left(\mathrm{n}_{11}, \mathrm{n}_{12}, \mathrm{n}_{21}, \mathrm{n}_{22}\right)$, and the lengths of the internal loops $\left(l_{11}, l_{12}, l_{21}, l_{22}\right)$. Exhaustive enumeration of all the possibilities for these 11 parameters for an $n$-nt chain requires a computational time scale on the order of $n^{11}$, which would be computationally unfeasible for long pseudoknot sequences.

If the internal/bulge loops in the helix stems $S_{1}$ and $S_{2}$ are flexible, $S_{1}$ and $S_{2}$ would be floppy, causing complications in the calculations of the loop $\left(\mathrm{L}_{1}\right.$ and $\left.\mathrm{L}_{2}\right)$ entropies. In order to make use of our entropy parameter table, which assumes rigid helix stems (Fig. 1A), we employ an approximation for the stems, as described below. The (internal/ bulge) loop tends to leave or enter a helix in a right-handed helical way, resulting in rigid conformations for a short internal/bulge loop. Therefore, for the purpose of the loop entropy calculation, we treat an internal/bulge loop as an effective helix of length $S_{1}^{\text {eff }}\left(S_{2}^{\text {eff }}\right)$ as determined by the following equations (see Fig. 5):

$$
S_{1}^{\text {eff }}=n_{11}+L_{12}+n_{12}, S_{2}^{\text {eff }}=n_{21}+L_{21}+n_{22} .
$$

In this way, we can read the entropy directly from the entropy table $\Delta \mathrm{S}\left(\mathrm{S}_{1}, \mathrm{~S}_{2}, \mathrm{~L}_{1}, \mathrm{~L}_{2}, \mathrm{~L}_{3}\right)$ with $\mathrm{S}_{1}$ and $\mathrm{S}_{2}$ substituted by the effective helix lengths $S_{1}^{\text {eff }}$ and $S_{2}^{\text {eff }}$ for stems $S_{1}$ and $S_{2}$, respectively. For pseudoknots without internal/bulge loops in the stems, $S_{1}^{\text {eff }}$ and $S_{2}^{\text {eff }}$ are equal to

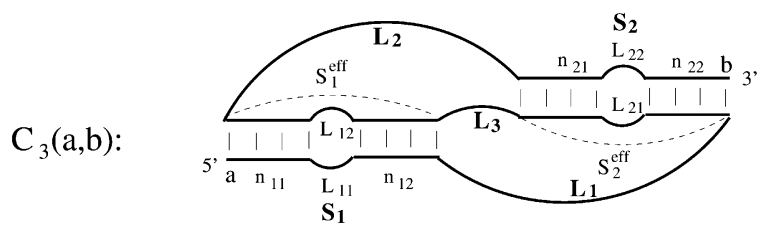

FIGURE 5. A general pseudoknotted structural element considered in the partition function calculation. 
the lengths of the original helices. An internal/bulge loop often causes bending of the stem $\left(S_{1}\right.$ or $\left.S_{2}\right)$. Through the above approximation, we replace a bent stem with a continuous helix for the purpose of loop entropy calculation. Our control tests show that the approximation causes minor errors: $\leq 5 \%$ and $15 \%$ in the entropy results with helix stems containing bulge loops of length $\leq 2 \mathrm{nt}$ and 3 nt, respectively (see details in the Supplemental Material).

For a loop $\left(\mathrm{L}_{1}\right.$ or $\left.\mathrm{L}_{2}\right)$ with nested helices (see Fig. 8A, below), we neglect the excluded volume effect from the nested helices and calculate the effective loop length as the number of helices plus the unpaired nucleotides outside the helices.

We separate out pseudoknot-containing structures from pseudoknot-free structures (secondary structures) in the partition function calculation. We compute the partition function $\mathrm{C}_{3}(a, b)$ for pseudoknot-containing structures from nucleotide a at the $5^{\prime}$ end to nucleotide $b$ at the $3^{\prime}$ end by enumerating all the possible values of helix stem lengths $S_{1}^{\text {eff }}$ and $S_{2}^{\text {eff }}$ and loop lengths $\mathrm{L}_{1}, \mathrm{~L}_{2}$, and $\mathrm{L}_{3}$ :

$$
\left.C_{3}(a, b)=\sum_{S_{1}^{\text {eff }}} \sum_{2}^{\text {eff }} \sum_{L_{1}} \sum_{L_{2}} \sum_{L_{3}} e^{-\Delta G\left(S_{1}^{\text {eff }}, S_{2}^{\text {eff }}, L_{1}, L_{2}, L_{3}\right)}\right) / k_{B} T,
$$

where $\Delta \mathrm{G}\left(S_{1}^{\text {eff }}, S_{2}^{\text {eff }}, \mathrm{L}_{1}, \mathrm{~L}_{2}, \mathrm{~L}_{3}\right)$ is the free energy for a given structure:

$$
\begin{aligned}
& \Delta G\left(S_{1}^{\text {eff }}, S_{2}^{\text {eff }}, L_{1}, L_{2}, L_{3}\right)=\Delta G_{\text {stem }}\left(S_{1}^{\text {eff }}\right)+\Delta G_{\text {stem }}\left(S_{2}^{\text {eff }}\right) \\
& \quad-T \Delta S\left(S_{1}^{\text {eff }}, S_{2}^{\text {eff }}, L_{1}, L_{2}, L_{3}\right) .
\end{aligned}
$$

We read out $\Delta S\left(S_{1}^{\text {eff }}, S_{2}^{\text {eff }}, L_{1}, L_{2}, L_{3}\right)$ from the entropy table. $\Delta \mathrm{G}_{\text {stem }}\left(S_{1}^{\text {eff }}\right)$ and $\Delta \mathrm{G}_{\text {stem }}\left(S_{2}^{\text {eff }}\right)$ are the folding free energy of the respective stems. $\Delta \mathrm{G}_{\text {stem }}\left(S^{\text {eff }}\right)$ for a stem $\left(\mathrm{S}_{1}\right.$ or $\left.\mathrm{S}_{2}\right)$ is computed from the local partition function for the stem:

$$
\Delta G_{\text {stem }}\left(S^{\text {eff }}\right)=-k_{B} T \ln \sum_{\text {internal } / \text { bulge loops }} e^{-\Delta G_{\text {stem }} / k_{B} T} .
$$

Here in the sum for stems with a given $S^{\text {eff }}$, we consider the presence and absence of an internal or bulge loop and the different sizes and positions of the loop. The free energy of the stem $\Delta G_{\text {stem }}$ in the above equation is the sum of the free energies for the base stacks and the loop in the stem, as determined by the nearest-neighbor model (Serra and Turner 1995; Cao and Chen 2005).

With the internal loops replaced by the effective helices in the loop entropy calculation, the conformational entropy for a general structure shown in Figure 5 is only dependent on five (instead of 11) parameters: $S_{1}^{\text {eff }}, S_{2}^{\text {eff }}, \mathrm{L}_{1}, \mathrm{~L}_{2}$, and $\mathrm{L}_{3}$. As shown in Equation 3, the computation for the partition function is now much more efficient, and the computational time scales as $\mathrm{n}^{5}$ instead of $\mathrm{n}^{11}$ for an $\mathrm{n}$-nt chain.

Using the recursive algorithm in Cao and Chen (2006b), we compute the total partition function $Q(a, b)$ for a chain from $a$ to $b$. From the conditional partition function $Q_{i j}$ for all the conformations that contain base pair $(i, j)$ between nucleotides $i$ and $j$, we compute the base-pairing probability $P_{i j}$ :

$$
P_{i j}=Q_{i j} / Q_{\text {tot }} .
$$

\begin{tabular}{|c|c|c|c|c|c|c|c|c|c|}
\hline Sequence ID & Length & Reference & Vfold & Hotknots & ILM & pknotsRE & STAR & Pknots-RG & NUPACK \\
\hline Bt-PrP & 45 & van Batenburg et al. (2000) & 0.42 & 0.41 & 0.83 & 0.5 & 0.33 & 0.33 & 0.41 \\
\hline BWYV & 28 & van Batenburg et al. (2000) & 1 & 1 & 0.88 & 1 & 1 & 1 & 1 \\
\hline Ec-PK1 & 30 & van Batenburg et al. (2000) & 1 & 1 & 0.36 & 1 & 0.36 & 1 & 1 \\
\hline Ec-PK4 & 52 & van Batenburg et al. (2000) & 0.84 & 0.68 & 0.52 & 0.68 & 0.68 & 0.68 & 1 \\
\hline Ec-S15 & 67 & van Batenburg et al. (2000) & 1 & 1 & 0.58 & 0.94 & 0.58 & 0.76 & 0.88 \\
\hline HIVRT32 & 35 & Tuerk et al. (1992) & 1 & 1 & 1 & 1 & 0.9 & 1 & 1 \\
\hline HIVRT322 & 35 & Tuerk et al. (1992) & 1 & 1 & 1 & 1 & 0.9 & 1 & 1 \\
\hline HIVRT33 & 35 & Tuerk et al. (1992) & 1 & 1 & 1 & 1 & 0 & 1 & 0.9 \\
\hline Hs-PrP & 45 & van Batenburg et al. (2000) & 0.45 & 0 & 0.27 & 0 & 0 & 0 & 0 \\
\hline LP-PK1 & 30 & van Batenburg et al. (2000) & 0.9 & 0.5 & 0.5 & 0.5 & 0.5 & 0.5 & 0.8 \\
\hline minimalIBV & 45 & Giedroc et al. (2000) & 0.94 & 0.94 & 0.88 & 0.94 & 0.88 & 0.94 & 0.94 \\
\hline MMTV & 34 & Giedroc et al. (2000) & 1 & 1 & 0.81 & 1 & 1 & 1 & 0.45 \\
\hline MMTV-vpk & 34 & Giedroc et al. (2000) & 1 & 1 & 0.54 & 1 & 1 & 1 & 1 \\
\hline pKA-A & 36 & Giedroc et al. (2000) & 1 & 1 & 1 & 1 & 1 & 1 & 1 \\
\hline SRV-1 & 38 & Giedroc et al. (2000) & 1 & 1 & 0 & 1 & 1 & 1 & 1 \\
\hline T2-gene32 & 33 & van Batenburg et al. (2000) & 1 & 1 & 0.58 & 1 & 1 & 1 & 1 \\
\hline T4-gene32 & 28 & van Batenburg et al. (2000) & 1 & 0.63 & 0.63 & 1 & 1 & 1 & 1 \\
\hline Tt-LSU-P3P7 & 65 & van Batenburg et al. (2000) & 0.85 & 0.95 & 0.8 & 0.9 & 0.6 & 0.85 & 0.95 \\
\hline Average & & & 0.91 & 0.84 & 0.68 & 0.86 & 0.71 & 0.84 & 0.85 \\
\hline
\end{tabular}

Here $Q_{\text {tot }}$ is the total partition function for all the possible structures. From $P_{i j}$ for all the possible $(i, j)$ 's, we can

TABLE 1. The sensitivity (SE) values for the structures predicted from seven different models

The tested sequences are adapted from Table 1 in Ren et al. (2005). Our Vfold model gives much improved sensitivity values for the 18 pseudoknot sequences. In the calculation, the temperature is $37^{\circ} \mathrm{C}$. Bold numbers show the highest accuracy. 
TABLE 2. The specificity (SP) values for the predicted structures from seven different models

\begin{tabular}{lclllllll}
\hline Sequence ID & Length & Vfold & Hotknots & ILM & pknotsRE & STAR & Pknots-RG & NUPACK \\
\hline Bt-PrP & 45 & 0.33 & 0.38 & $\mathbf{0 . 7 6}$ & 0.5 & 0.26 & 0.26 & 0.38 \\
BWYV & 28 & $\mathbf{1}$ & $\mathbf{1}$ & $\mathbf{1}$ & $\mathbf{1}$ & $\mathbf{1}$ & $\mathbf{1}$ & $\mathbf{1}$ \\
EC-PK1 & 30 & 0.92 & $\mathbf{1}$ & 0.44 & $\mathbf{1}$ & 0.5 & $\mathbf{1}$ & $\mathbf{1}$ \\
EC-PK4 & 52 & $\mathbf{1}$ & $\mathbf{1}$ & 0.58 & 0.92 & $\mathbf{1}$ & $\mathbf{1}$ & $\mathbf{1}$ \\
EC-S15 & 67 & $\mathbf{1}$ & 0.73 & 0.47 & 0.64 & 0.62 & 0.68 & 0.71 \\
HIVRT32 & 35 & $\mathbf{1}$ & $\mathbf{1}$ & $\mathbf{1}$ & $\mathbf{1}$ & $\mathbf{1}$ & $\mathbf{1}$ & $\mathbf{1}$ \\
HIVRT322 & 35 & $\mathbf{1}$ & $\mathbf{1}$ & $\mathbf{1}$ & $\mathbf{1}$ & $\mathbf{1}$ & $\mathbf{1}$ & $\mathbf{1}$ \\
HIVRT33 & 35 & $\mathbf{1}$ & $\mathbf{1}$ & $\mathbf{1}$ & $\mathbf{1}$ & 0 & $\mathbf{1}$ & $\mathbf{1}$ \\
Hs-PrP & 45 & $\mathbf{0 . 5}$ & 0 & 0.27 & 0 & 0 & 0 & 0 \\
LP-PK1 & 30 & 0.9 & $\mathbf{1}$ & 0.71 & 0.83 & $\mathbf{1}$ & $\mathbf{1}$ & $\mathbf{1}$ \\
minimalIBV & 45 & $\mathbf{0 . 9 4}$ & 0.88 & 0.88 & $\mathbf{0 . 9 4}$ & 0.93 & $\mathbf{0 . 9 4}$ & $\mathbf{0 . 9 4}$ \\
MMTV & 34 & $\mathbf{1}$ & 0.91 & 0.81 & 0.91 & 0.91 & 0.91 & 0.5 \\
MMTV-vpk & 34 & 0.92 & 0.91 & 0.54 & 0.91 & 0.91 & 0.91 & $\mathbf{1}$ \\
pKA-A & 36 & $\mathbf{1}$ & 0.92 & 0.92 & 0.92 & 0.92 & 0.92 & 0.92 \\
SRV-1 & 38 & $\mathbf{1}$ & 0.91 & 0 & 0.91 & 0.91 & 0.91 & 0.91 \\
T2-gene32 & 33 & $\mathbf{1}$ & $\mathbf{1}$ & 0.7 & $\mathbf{1}$ & $\mathbf{1}$ & $\mathbf{1}$ & $\mathbf{1}$ \\
T4-gene32 & 28 & $\mathbf{1}$ & 0.87 & $\mathbf{1}$ & $\mathbf{1}$ & $\mathbf{1}$ & $\mathbf{1}$ & $\mathbf{1}$ \\
Tt-LSU-P3P7 & 65 & 0.85 & $\mathbf{1}$ & 0.69 & 0.85 & 0.75 & $\mathbf{1}$ & $\mathbf{1}$ \\
Average & & $\mathbf{0 . 9 1}$ & 0.86 & 0.71 & 0.85 & 0.76 & 0.86 & 0.85 \\
\hline
\end{tabular}

The tested sequences are adapted from Table 1 in Ren et al. (2005). Our Vfold model gives much improved specificity values for the 18 pseudoknot sequences. Bold numbers show the highest accuracy.
(Zwieb et al. 1999), and Simian retrovirus type-1 (SRV-1) (ten Dam et al. 1995) forms a pseudoknot that promotes the ribosomal frameshifting. For the two H-type pseudoknots, our Vfold model gives the highest $\mathrm{SE}$ value (see Fig. 6A,B; Table 1). We note that the ILM model gives a false prediction for the SRV-1 pseudoknot. The failure of the ILM model may be due to the fact that the model does not account for the loop entropy for pseudoknots.

\section{VMV pseudoknot}

From a recent biochemical study, Pennell and Brierley and colleagues found that the stimulatory RNA for VMV frameshifting forms a pseudoknot structure (Pennell et al. 2008) instead of a stem-loop strucpredict the stable structures and the equilibrium folding pathways.

\section{STRUCTURAL PREDICTIONS}

\section{Comparison with other models}

We measure the accuracy of structure predictions by two parameters: (1) the sensitivity parameter SE, defined as the ratio between the number of correctly predicted base pairs and number of the base pairs in the experimentally determined structure; and (2) the specificity parameter SP, defined as the ratio between the number of correctly predicted base pairs and the total number of predicted base pairs. Our tests for structural predictions indicate that the model developed here gives better results than other models that we have tested (see Tables 1,2). Specifically, our model gives the highest SE value for 15 sequences among the total 18 sequences, and the highest SP value for 13 sequences. In addition, our model gives higher overall average SE (0.91) and SP (0.91) than other models.

\section{H-type pseudoknot}

LP-PK1 and SRV-1 are two H-type pseudoknots. LP-PK1 is a PK1 domain of Legionella pneumophila tmRNA ture. Moreover, the pseudoknot is quite unique because it contains a long interhelix loop. We perform the structural prediction for this 67-nt RNA. Figure 7A shows that the predicted structure agrees exactly with the experimental structure, with SE and SP values both equal to 1.

\section{R2 retrotransposon pseudoknot}

The $5^{\prime}$ header of the $\mathrm{R} 2$ retrotransposon controls the $\mathrm{R} 2$ protein binding and cleavage of the DNA target (Christensen
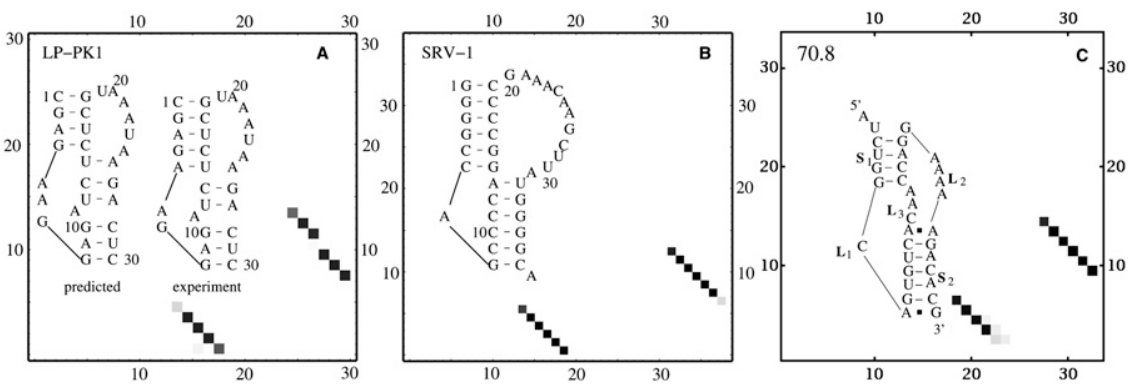

FIGURE 6. The predicted structures for three pseudoknots. (A) For LP-PK1, Hotknots (Ren et al. 2005), ILM (Ruan et al. 2004), pknotsRE (Rivas and Eddy 1999), STAR (Gultyaev et al. 1995), and pknots-RG (Reeder and Giegerich 2004) all give poor predictions for the structure $(\mathrm{SE}=0.5)$. NUPACK (Dirks and Pierce 2003) gives a relatively high SE value $(\mathrm{SE}=0.8)$. Our Vfold model gives the highest accuracy with SE $=0.9$. (B) For the SRV-1 pseudoknot, the ILM model fails to predict the native structure of the SRV-1 pseudoknot. (C) For the 70.8 anti-HIV aptamer, we predict a pseudoknot with a 3-nt interhelix loop. In the calculations, the temperature is $37^{\circ} \mathrm{C}$ for $A$ and $B$ and $20^{\circ} \mathrm{C}$ for $C$ according to the experimental condition (Held et al. 2006a,b). Also shown in the figures are the density plot for the base-pairing probability $P_{i j}$ (Equation 4$)$. In the density plots, the horizontal and vertical axes denote the indices of the nucleotides $i$ and $j$. 

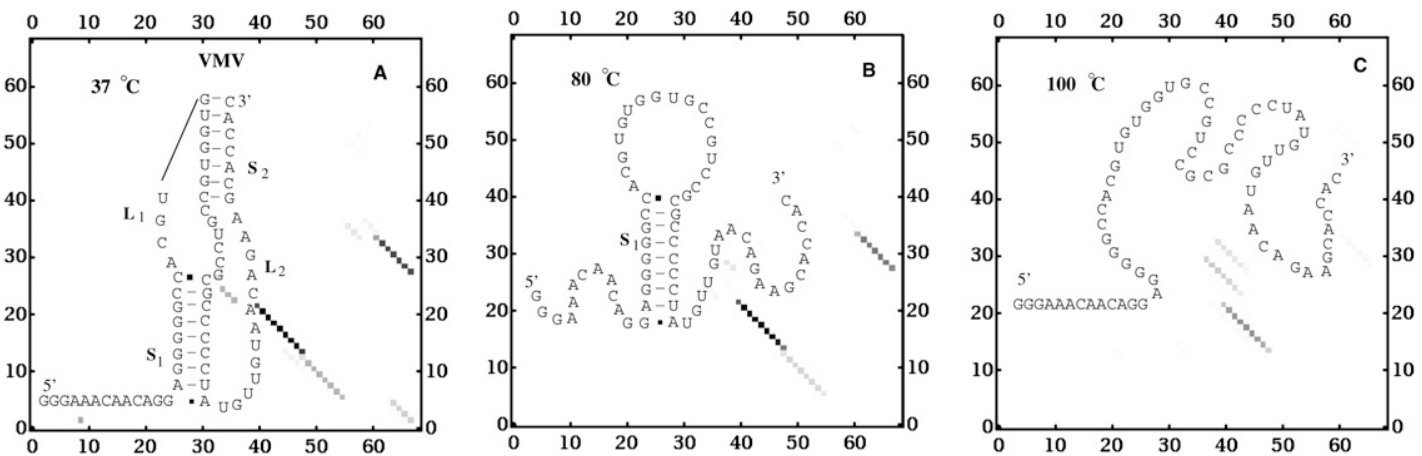

FIGURE 7. The density plots and the predicted structures for the 67-nt $5^{\prime}$ VMV pseudoknot at different temperatures. Stem $S_{1}$ is the most stable stem, which is the last one to be unzipped. At $T=37^{\circ} \mathrm{C}$, our predicted structure shows the highest $\mathrm{SE}=1.0$ and $\mathrm{SP}=1.0$ as tested against the experimental structure (Pennell et al. 2008).

et al. 2006). Based on the NMR spectra and computational models (Hart et al. 2008), Hart and Turner and colleagues found a knotted structure in the 74-nt header of the $\mathrm{R} 2$ retrotransposon. In this study, we use the Vfold model developed here to predict the secondary structure for the 74-nt header. Figure 8A shows our predicted structure. The predicted structure is a pseudoknot with four stems. All four stems have been found in the experiments (Hart et al. 2008). The predicted structure shows a high accuracy with $\mathrm{SE}=1.0$ and $\mathrm{SP}=1.0$. In the calculation, we have added the base-stacking energy for the WatsonCrick base pairs between nucleotides 48CG49 and 62CG63 (see the dashed lines in Fig. 8A). This tertiary interaction has been confirmed in previous NMR measurement (Ferré-D’Amaré et al. 1998).

\section{Anti-HIV RNA aptamer}

Recently experiments suggested that the interhelix loop may be essential for efficient ribosomal frameshifting (Brierley et al. 2008; Giedroc and Cornish 2008). Moreover, previous experimental studies on the anti-HIV RNA aptamer (Held et al. 2006a,b) suggested that the interhelix loop, which causes flexible helix orientations in the pseudoknot aptamers, may play an important functional role in accommodating aptamer binding to the HIV reverse transcriptase. For example, for an aptamer (labeled as 70.8 according to the notations used in the literature) (Held et al. 2006a,b), the proposed native structure contains a 3-nt interhelix loop.
The predicted structure from our model (Fig. 6C), indeed, shows a 3-nt interhelix loop. The structure has a high accuracy of $\mathrm{SE}=0.9$ and $\mathrm{SP}=1.0$ if we treat the experimentally proposed structure (Held et al. 2006a,b) as the "experimental" structure.
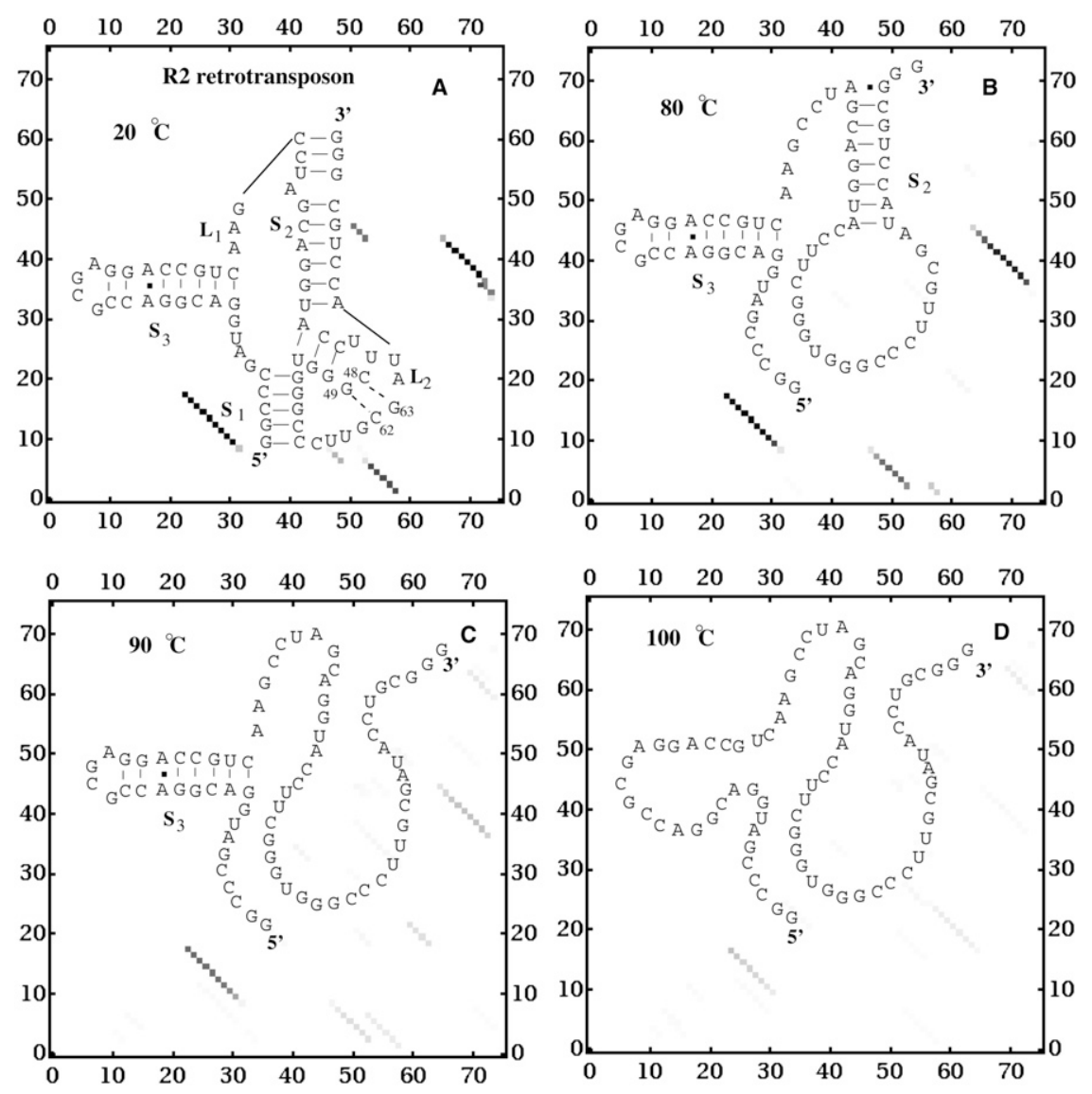

FIGURE 8. The density plots and the predicted structures for the 74-nt $5^{\prime}$ header of an R2 retrotransposon at different temperatures. Stem $\mathrm{S}_{3}$ is the most stable stem, which is the last one to be unzipped. At room temperature, the predicted structure shows the highest $\mathrm{SE}=1.0$ and $\mathrm{SP}=1.0$ as tested against the experimental NMR structure (Hart et al. 2008). 


\section{FOLDING THERMODYNAMICS}

\section{The 70.8 aptamer}

As the temperature increases, stems $S_{1}$ and $S_{2}$ of the pseudoknot (see Fig. 6A) is disrupted at nearly the same temperature. At $T=80^{\circ} \mathrm{C}$, both stems are unfolded. Thus, stems $S_{1}$ and $S_{2}$ have the comparable thermal stability.

\section{VMV pseudoknot}

A recent combined biochemical and NMR experiment (Pennell et al. 2008) showed that the VMV pseudoknot contains a 6-nt interhelix loop. Our predicted unfolding pathway suggests that at $T=80^{\circ} \mathrm{C}$, stem $\mathrm{S}_{2}$ is the first stem to be unzipped, and stem $S_{1}$ is the last one to be unzipped (see Fig. $7 \mathrm{~B}, \mathrm{C})$. Our prediction agrees with the experimental finding (Pennell et al. 2008), which suggested that $S_{1}$ is the most stable and $S_{2}$ is disrupted at a temperature around $76.8^{\circ} \mathrm{C}$.

\section{R2 retrotransposon pseudoknot}

The native structure of the $\mathrm{R} 2$ retrotransposon pseudoknot contains four stems (Fig. 8A). The structure shows high stability against temperature increase. At $T=80^{\circ} \mathrm{C}$, stem $\mathrm{S}_{1}$ is the first stem to be unfolded, resulting in an intermediate state that contains stem $\mathrm{S}_{3}$ and a partially unfolded stem $\mathrm{S}_{2}$. As the temperature is further increased to $90^{\circ} \mathrm{C}$, stem $S_{2}$ becomes completely unzipped since the hairpin with $\mathrm{S}_{2}$ is destabilized by the large loop. Stem $S_{3}$ is the most robust stem and is the last stem to be unzipped. The melting temperature for $S_{3}$ is $\sim 100^{\circ} \mathrm{C}$.

\section{SUMMARY}

In summary, we have developed a new virtual-bond-based model (Vfold) for general RNA pseudoknots with interhelix loops. The model allows an accurate treatment for the loop-helix excluded volume interactions and rigorous calculations for the conformational entropy for general pseudoknotted folds. Tests against other existing models suggest that this new model gives improved predictions for the native structures, with average sensitivity and specificity measures of the accuracy equal to 0.91 and 0.91 , respectively. We attribute the improved accuracy to the rigorous conformational entropy parameters. For any given RNA sequence, the model enables predictions for not only the native structures, but also the folding stabilities and equilibrium folding pathways. Despite the success of this new model, it has several limitations that should be removed in future model development. First, the model does not treat possible noncanonical interactions such as base triple interactions between loops and stems and noncanonical basepairing between loop nucleotides. These interactions can be biologically important for more complex pseudoknotted structures (Cornish et al. 2005; Theimer et al. 2005). Moreover, ions, especially $\mathrm{Mg}^{2+}$ ions, can play an important role in loop entropy and the global folding stability of pseudoknots (Chen 2008; Tan and Chen 2008).

\section{SUPPLEMENTAL MATERIAL}

Supplemental material can be found at http://www.rnajournal.org.

\section{ACKNOWLEDGMENTS}

We thank Professor Donald H. Burke for useful discussions. The research was supported by NIH through grant GM063732 (to S.-J.C.). Most of the computations involved in this research were performed on the HPC resources at the University of Missouri Bioinformatics Consortium (UMBC).

Received October 21, 2008; accepted January 10, 2009.

\section{REFERENCES}

Arnott, S. and Hukins, D.W.L. 1972. Optimized parameters for RNA double-helices. Biochem. Biophys. Res. Commun. 48: 1392-1399.

Bon, M., Vernizzi, G., Orland, H., and Zee, A. 2008. Topological classification of RNA structures. J. Mol. Biol. 379: 900-911.

Brierley, I., Digard, P., and Inglis, S.C. 1989. Characterization of an efficient coronavirus ribosomal frameshifting signal: requirement for an RNA pseudoknot. Cell 57: 537-547.

Brierley, I., Pennell, S., and Gilbert, R.J.C. 2007. Viral RNA pseudoknots: Versatile motifs in gene expression and replication. Nat. Rev. Microbiol. 5: 598-610.

Brierley, I., Gilbert, R.J.C., and Pennell, S. 2008. RNA pseudoknots and the regulation of protein synthesis. Biochem. Soc. Trans. 36: 684-689.

Burke, D.H., Scates, L., Andrews, K., and Gold, L. 1996. Bent pseudoknots and novel RNA inhibitors of type 1 human immunodeficiency virus (HIV-1) reverse transcriptase. J. Mol. Biol. 264: 650-666.

Cao, S. and Chen, S.-J. 2005. Predicting RNA folding thermodynamics with a reduced chain representation model. RNA 11: 1884-1897.

Cao, S. and Chen, S.-J. 2006a. Free-energy landscapes of RNA/RNA complexes: With applications to snRNA complexes in spliceosomes. J. Mol. Biol. 357: 292-312.

Cao, S. and Chen, S.-J. 2006b. Predicting RNA pseudoknot folding thermodynamics. Nucleic Acids Res. 34: 2634-2652.

Cao, S. and Chen, S.-J. 2008. Predicting ribosomal frameshifting efficiency. Phys. Biol. 5: 016002. doi: 10.1088/1478-3975/5/1/016002.

Chen, S.-J. 2008. RNA folding: Conformational statistics, folding kinetics, and ion electrostatics. Annu. Rev. Biophys. 37: 197-214.

Chen, S.-J. and Dill, K.A. 2000. RNA folding energy landscapes. Proc. Natl. Acad. Sci. 97: 646-651.

Chen, X.Y., Kang, H.S., Shen, L.X., Chamorro, M., Varmus, H.E., and Tinoco Jr., I. 1996. A characteristic bent conformation of RNA pseudoknots promotes -1 frameshifting during translation of retroviral RNA. J. Mol. Biol. 260: 479-483.

Chen, J.L., Blasco, M.A., and Greider, C.W. 2000. Secondary structure of vertebrate telomerase RNA. Cell 100: 503-514.

Chen, X., He, S., Zhang, F., Wang, Z., Chen, R., and Gao, W. 2008. FlexStem: Improving predictions of RNA secondary structures with pseudoknots by reducing the search space. Bioinformatics 24: 1994-2001.

Chu, V.B. and Herschlag, D. 2008. Unwinding RNA's secrets: Advances in the biology, physics, and modeling of complex RNAs. Curr. Opin. Struct. Biol. 18: 305-314. 
Comolli, L.R., Smirnov, I., Xu, L., Blackburn, E.H., and James, T.L. 2002. A molecular switch underlies a human telomerase disease. Proc. Natl. Acad. Sci. 99: 16998-17003.

Cornish, P.V., Hennig, M., and Giedroc, D.P. 2005. A loop 2 cytidinestem 1 minor groove interaction as a positive determinant for pseudoknot-stimulated -1 ribosomal frameshifting. Proc. Natl. Acad. Sci. 102: 12694-12699.

Christensen, S.M., Ye, J.Q., and Eickbush, T.H. 2006. RNA from the 5' end of the R2 retrotransposon controls $\mathrm{R} 2$ protein binding to and cleavage of its DNA target site. Proc. Natl. Acad. Sci. 103: 17602-17607.

Deiman, B.A., Kortlever, R.M., and Pleij, C.W. 1997. The role of the pseudoknot at the $3^{\prime}$ end of turnip yellow mosaic virus RNA in minus-strand synthesis by the viral RNA-dependent RNA polymerase. J. Virol. 71: 5990-5996.

Ding, Y. 2006. Statistical and Bayesian approaches to RNA secondary structure prediction. RNA 12: 323-331.

Dirks, R.M. and Pierce, N.A. 2003. A partition function algorithm for nucleic acid secondary structure including pseudoknots. J. Comput. Chem. 24: 1664-1677.

Duarte, C.M. and Pyle, A.M. 1998. Stepping through an RNA structure: A novel approach to conformational analysis. J. Mol. Biol. 284: 1465-1478.

Ferré-D’Amaré, A.R., Zhou, K.H., and Doudna, J.A. 1998. Crystal structure of a hepatitis delta virus ribozyme. Nature 395: 567-574.

Flory, P.J. 1969. Statistical mechanics of chain molecules. Wiley, New York.

Giedroc, D.P. and Cornish, P.V. 2008. Frameshifting RNA pseudoknots: Structure and mechanism. Virus Res. (in press). doi: 10.1016/ j.virusres.2008.06.008.

Giedroc, D.P., Theimer, C.A., and Nixon, P.L. 2000. Structure, stability and function of RNA pseudoknots involved in stimulating ribosomal frameshifting. J. Mol. Biol. 298: 167-185.

Gultyaev, A.P., van Batenburg, F.H.D., and Pleij, C.W.A. 1995. The computer simulation of RNA folding pathways using a genetic algorithm. J. Mol. Biol. 250: 37-51.

Gultyaev, A.P., Van Batenburg, F.H.D., and Pleij, C.W.A. 1999. An approximation of loop free-energy values of RNA H-pseudoknots. RNA 5: 609-617.

Hansen, T.M., Reihani, S.N.S., Oddershede, L.B., and Sørensen, M.A. 2007. Correlation between mechanical strength of messenger RNA pseudoknots and ribosomal frameshifting. Proc. Natl. Acad. Sci. 104: $5830-5835$.

Hart, J.M., Kennedy, S.D., Mathews, D.H., and Turner, D.H. 2008. NMR-assisted prediction of RNA secondary structure: Identification of a probable pseudoknot in the coding region of an R2 retrotransposon. J. Am. Chem. Soc. 130: 10233-10239.

Held, D.M., Kissel, J.D., Saran, D., Michalowski, D., and Burke, D.H. 2006a. Differential susceptibility of HIV-1 reverse transcriptase to inhibition by RNA aptamers in enzymatic reactions monitoring specific steps during genome replication. J. Biol. Chem. 281: 25712-25722

Held, D.M., Kissel, J.D., Patterson, J.T., Nickens, D.G., and Burke, D.H. 2006b. HIV-1 inactivation by nucleic acid aptamers. Front. Biosci. 11: 89-112.

Hofacker, I.L. 2003. Vienna RNA secondary structure server. Nucleic Acids Res. 31: 3429-3431.

Huang, X. and Ali, H. 2007. High sensitivity RNA pseudoknot prediction. Nucleic Acids Res. 35: 656-663.

Isambert, H. and Siggia, E.D. 2000. Modeling RNA folding paths with pseudoknots: Application to hepatitis delta virus ribozyme. Proc. Natl. Acad. Sci. 97: 6515-6520.

Jabbari, H., Condon, A., and Zhao, S. 2008. Novel and efficient RNA secondary structure prediction using hierarchical folding. $J$. Comput. Biol. 15: 139-163.

Jossinet, F., Ludwig, T.E., and Westhof, E. 2007. RNA structure: Bioinformatic analysis. Curr. Opin. Microbiol. 10: 279-285.

Kopeikin, Z. and Chen, S.J. 2006. Folding thermodynamics of pseudoknotted chain conformations. J. Chem. Phys. 124: 154903. doi: 10.1063/1.2188940.
Li, P.T.X., Vieregg, J., and Tinoco Jr., I. 2008. How RNA unfolds and refolds. Annu. Rev. Biochem. 77: 77-100.

Mathews, D.H. and Turner, D.H. 2006. Prediction of RNA secondary structure by free-energy minimization. Curr. Opin. Struct. Biol. 16: $270-278$.

Mathews, D.H., Sabina, J., Zuker, M., and Turner, D.H. 1999. Expanded sequence dependence of thermodynamic parameters improves prediction of RNA secondary structure. J. Mol. Biol. 288: 911-940.

McCaskill, J.S. 1990. The equilibrium partition function and base-pair binding probabilities for RNA secondary structure. Biopolymers 29: $1105-1119$

Metzler, D. and Nebel, M.E. 2008. Predicting RNA secondary structures with pseudoknots by MCMC sampling. J. Math. Biol. 56: $161-181$.

Michiels, P.J.A., Versleijen, A.A.M., Verlaan, P.W., Pleij, C.W.A., Hilbers, C.W., and Heus, H.A. 2001. Solution structure of the pseudoknot of SRV-1 RNA, involved in ribosomal frameshifting. $J$. Mol. Biol. 310: 1109-1123.

Murray, L.J., Arendall III., W.B., Richardson, D.C., and Richardson, J.S. 2003. RNA backbone is rotameric. Proc. Natl. Acad. Sci. 100: 13904-13909.

Murthy, V.L., Srinivasan, R., Draper, D.E., and Rose, G.D. 1999. A complete conformational map for RNA. J. Mol. Biol. 291: 313327.

Namy, O., Moran, S.J., Stuart, D.I., Gilbert, R.J., and Brierley, I. 2006. A mechanical explanation of RNA pseudoknot function in programmed ribosomal frameshifting. Nature 441: 244-247.

Nussinov, R. and Jacobson, A.B. 1980. Fast algorithm for predicting the secondary structure of single-stranded RNA. Proc. Natl. Acad. Sci. 77: 6909-6913.

Nussinov, R., Pieczenik, G., Griggs, J., and Kleitman, D. 1978. Algorithms for loop matchings. SIAM Rev. Soc. Ind. Appl. Math. 35: $68-82$.

Olson, W.K. 1980. Configurational statistics of polynucleotide chains: An updated virtual bond model to treat effects of base stacking. Macromolecules 13: 721-728.

Olson, W.K. and Flory, P.J. 1972. Spatial configurations of polynucleotide chains. I, Steric interactions in polyribonucleotides: A virtual bond model. Biopolymers 11: 1-23.

Pennell, S., Manktelow, E., Flatt, A., Kelly, G., Smerdon, S.J., and Brierley, I. 2008. The stimulatory RNA of the Visna-Maedi retrovirus ribosomal frameshifting signal is an unusual pseudoknot with an interstem element. RNA 14: 1366-1377.

Perrotta, A.T. and Been, M.D. 1991. A pseudoknot-like structure required for efficient self-cleavage of hepatitis delta-virus RNA. Nature 350: 434-436.

Plant, E.P., Jacobs, K.L., Harger, J.W., Meskauskas, A., Jacobs, J.L., Baxter, J.L., Petrov, A.N., and Dinman, J.D. 2003. The $9 \AA$ solution: How mRNA pseudoknots promote efficient programmed -1 ribosomal frameshifting. RNA 9: 168-174.

Reeder, J. and Giegerich, R. 2004. Design, implementation, and evaluation of a practical pseudoknot folding algorithm based on thermodynamics. BMC Bioinformatics 5: 104. doi: 10.1186/14712105-5-104.

Reeder, J., Hochsmann, M., Rehmsmeier, M., Voss, B., and Giegerich, R. 2006. Beyond Mfold: Recent advances in RNA bioinformatics. J. Biotechnol. 124: 41-55.

Ren, J., Rastegari, B., Condon, A., and Hoos, H.H. 2005. HotKnots: Heuristic prediction of RNA secondary structures including pseudoknots. RNA 11: 1494-1504.

Richardson, J.S., Schneider, B., Murray, L.W., Kapral, G.J., Immormino, R.M., Headd, J.J., Richardson, D.C., Ham, D., Hershkovits, E., Williams, L.D., et al. 2008. RNA backbone: Consensus all-angle conformers and modular string nomenclature (an RNA Ontology Consortium contribution). RNA 14: 465-481.

Rivas, E. and Eddy, S.R. 1999. A dynamic programming algorithm for RNA structure prediction including pseudoknots. J. Mol. Biol. 285: 2053-2068. 
Ruan, J., Stormo, G.D., and Zhang, W. 2004. An iterated loop matching approach to the prediction of RNA secondary structures with pseudoknots. Bioinformatics 20: 58-66.

Schultes, E.A. and Bartel, D.P. 2000. One sequence, two ribozymes: Implications for the emergence of new ribozyme folds. Science 289: 448-452.

Schuster, P. 2006. Prediction of RNA secondary structures: From theory to models and real molecules. Rep. Prog. Phys. 69: 1419-1477.

Serra, M.J. and Turner, D.H. 1995. Predicting thermodynamic properties of RNA. Methods Enzymol. 259: 242-261.

Shapiro, B.A., Yingling, Y.G., Kasprzak, W., and Bindewald, E. 2007. Bridging the gap in RNA structure prediction. Curr. Opin. Struct. Biol. 17: 157-165.

Somogyi, P., Jenner, A.J., Brierley, I., and Inglis, S.C. 1993. Ribosomal pausing during translation of an RNA pseudoknot. Mol. Cell. Biol. 13: $6931-6940$.

Sperschneider, J. and Datta, A. 2008. KnotSeeker: Heuristic pseudoknot detection in long RNA sequences. RNA 14: 630-640.

Staple, D.W. and Butcher, S.E. 2005. Pseudoknots: RNA structures with diverse functions. PLoS Biol. 3: e213. doi: 10.1371/journal.pbio.0030213.

Su, L., Chen, L., Egli, M., Berger, J.M., and Rich, A. 1999. Minor groove RNA triplex in the crystal structure of a ribosomal frameshifting viral pseudoknot. Nat. Struct. Biol. 6: 285-292.

Tan, Z.J. and Chen, S.-J. 2008. Salt dependence of nucleic acid hairpin stability. Biophys. J. 95: 738-752.

Tanner, N.K., Schaff, S., Thill, G., Petitkoskas, E., Craindenoyelle, A.M., and Westhof, E. 1994. A three-dimensional model of hepatitis delta virus ribozyme based on biochemical and mutational analyses. Curr. Biol. 4: 488-498.

ten Dam, E., Verlaan, P.W.G., and Pleij, C.W.A. 1995. Analysis of the role of the pseudoknot component in the SRV-1 gag-pro ribosomal frameshift signal: Loop lengths and stability of the stem regions. RNA 1: 146-154.
Theimer, C.A., Blois, C.A., and Feigon, J. 2005. Structure of the human telomerase RNA pseudoknot reveals conserved tertiary interactions essential for function. Mol. Cell 17: 671-682.

Tuerk, C., MacDougal, S., and Gold, L. 1992. RNA pseudoknots that inhibit human immunodeficiency virus type 1 reverse transcriptase. Proc. Natl. Acad. Sci. 89: 6988-6992.

van Batenburg, F.H.D., Gultyaev, A.P., Pleij, C.W.A., Ng, J., and Oliehoek, J. 2000. Pseudobase: A database with RNA pseudoknots. Nucleic Acids Res. 28: 201-204.

van Belkum, A., Abrahams, J., Pleij, C., and Bosch, L. 1985. Five pseudoknots are present at the 204 nucleotides long $3^{\prime}$ noncoding region of tobacco mosaic virus RNA. Nucleic Acids Res. 13: 76737686.

Wadley, L.M., Keating, K.S., Duarte, C.M., and Plye, A.M. 2007. Evaluating and learning from RNA pseudotorsional space: Quantitative validation of a reduced representation for RNA structure. J. Mol. Biol. 372: 942-957.

Walter, A.E. and Turner, D.H. 1994. Sequence dependence of stability for coaxial stacking of RNA helixes with Watson-Crick base paired interfaces. Biochemistry 33: 12715-12719.

Williams Jr., A.L. and Tinoco Jr., I. 1986. A dynamic programming algorithm for finding alternative RNA secondary structures. Nucleic Acids Res. 14: 299-315.

Zhang, W.B. and Chen, S.J. 2001. A three-dimensional statistical mechanical model of folding double-stranded chain molecules. J. Chem. Phys. 114: 7669-7681.

Zhang, J., Lin, M., Chen, R., Wang, W., and Liang, J. 2008. Discrete state model and accurate estimation of loop entropy of RNA secondary structures. J. Chem. Phys. 128: 125107. doi: 10.1063/ 1.2895050 .

Zuker, M. 1989. On finding all suboptimal foldings of an RNA molecule. Science 244: 48-52.

Zwieb, C., Wower, I., and Wower, J. 1999. Comparative sequence analysis of tmRNA. Nucleic Acids Res. 27: 2063-2071. 

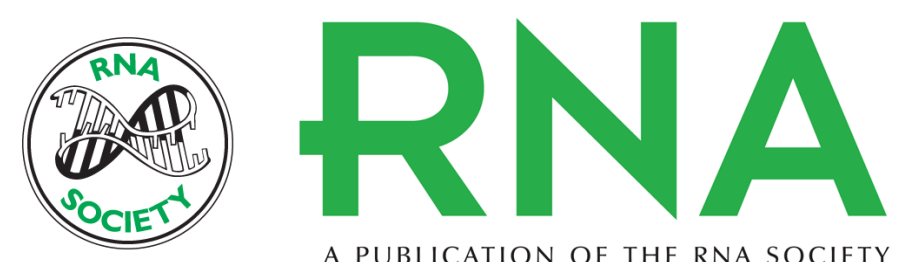

A PUBLICATION OF THE RNA SOCIETY

\section{Predicting structures and stabilities for H-type pseudoknots with interhelix loops}

Song Cao and Shi-Jie Chen

RNA 2009 15: 696-706 originally published online February 23, 2009

Access the most recent version at doi:10.1261/rna.1429009

\section{Supplemental http://rnajournal.cshlp.org/content/suppl/2009/02/23/rna.1429009.DC1 \\ Material}

References This article cites 81 articles, 22 of which can be accessed free at: http://rnajournal.cshlp.org/content/15/4/696.full.html\#ref-list-1

\section{License}

Email Alerting Receive free email alerts when new articles cite this article - sign up in the box at the Service top right corner of the article or click here. 